\title{
El Trastorno del Espectro Autista (TEA) y el uso de las Tecnologías de la información y comunicación (TIC)
}

Recibido: 4 de julio de 2019 / Revisado: 18 de julio de 2019

Aceptado: 5 de noviembre de 2019 / Publicado: 20 de diciembre de 2019

DESIRÉE DOMÍNGUEZ BARQUERO

Universidad de Granada, España desireedoba@correo.ugr.es

http://dx.doi.org/10.24310/IJNE2.2.2019.7447

\section{RESUMEN}

La temática que aborda esta investigación se centra en el Trastorno del Espectro Autista o TEA y el uso de las Tecnologías de la Comunicación y la Información (TIC) en este trastorno. En primer lugar, vamos a realizar una breve introducción sobre el tema en cuestión. El estudio se fundamenta en la explicación de que es el TEA, a partir de un recorrido histórico sobre el trastorno, luego se dará una definición según el DSM-V y pasaremos a la tipología sobre el TEA a partir del CIE-10-ES. Luego se hablará de la intervención educativa que se lleva a cabo con alumnado con TEA y que y quienes son necesarios para realizar esta evaluación de una manera más exacta. Explicaremos algunos de los elementos que se usan para la intervención y posteriormente hablaremos de la intervención educativa de las TIC en alumnado con TEA. Explicando primeramente que son las TIC, la evolución que ha tenido a lo largo de la historia y que es necesario tener un mínimo de conocimientos para poder usarlas. Y por último se explicará el uso y los beneficios que tienen para alumnado con TEA.

\section{ABSTRACT}

Autism Spectrum Disorder (ASD) and the use of Information and Communication Technologies (ICT)

The topic addressed by this research focuses on Autism Spectrum Disorder or ASD and the use of Communication and Information Technologies (ICTs) in this disorder. First of all there will be a brief introduction on the subject at hand. The study is based on the explanation that it is ASD, from a historical tour on the disorder, then a definition according to the DSM-V will be given and we will move to the type on ASD from ICD-10-ES.Then we will talk about the educational intervention that takes place with students with ASD and who are necessary to carry out this evaluation in a more accurate way. We will explain some of the elements used for intervention and then talk about the educational intervention of ICT in students with ASD. First explaining that it is ICT, the evolution that has had throughout history and that it is necessary to have a minimum of knowledge in order to use them. And finally, the use and benefits they have for students with ASD will be explained. 
Palabras Clave: Autismo, Trastorno espectro autista, TEA, Intervención educativa, Tecnologías de la información y comunicación, TIC, Educación infantil.
Keywords: Autism, Autism Spectrum Disorder, ASD, Educational Intervention, Information and Communication Technologies, ICT, Children's Education.

\section{Introducción}

En los últimos años se ha investigado mucho sobre la incorporación de las Tecnologías de la información y comunicación (TIC) y los beneficios que incorporan en el ámbito de la educación, tanto en la enseñanza por parte del profesorado o el equipo docente, como en el aprendizaje general en el aula y en el trabajo con alumnado con TEA. Se ha podido comprobar que el uso de las TIC es positivo en el proceso de Enseñanza-Aprendizaje, ya que favorece que se pueda realizar un aprendizaje dependiendo del ritmo, maduración y nivel cognitivo, motor, etc.; que tiene cada alumno o alumna con TEA, ya que como es sabido no cada persona con Trastorno del Espectro Autista tiene las mismas características ni las mismas carencias, por ello es necesario un plan individualizado en el que podamos tratar con sus intereses, motivaciones, ritmos y características. Por ello el uso de las TIC nos da vía libre a la hora de poder obtener este tipo de enseñanzas, ya que tiene gran versatilidad, flexibilidad y se puede adaptar según las necesidades de cada persona o de cada momento. Por otro lado, vemos como el uso de las TIC hace que los niños y niñas con TEA estén más motivados y favorezca su aprendizaje con mayor facilidad ya que lo refuerzan de manera agradable, divertida y atrayente, ya que el alumnado con TEA tiene una serie de cualidades visuales para el procesamiento de la información que las TIC lo facilitan en gran medida.

También debemos tener en cuenta que el alumnado con TEA tiene problemas a la hora de tener cambios en su entorno, por ello con ayuda de las TIC fomentamos que se pueda estructurar y organizar el entorno de interacción y así evitar la conflictividad que ello produce e intentar que el aula y sus actividades sean predecibles para el alumnado.

Con ellas también a parte de trabajar la estructuración y organización del horario y de la entrada de maestros y maestras al aula, también fomentamos una mayor autonomía ya que potencian que ellos solos interactúen y formen parte activa de su proceso de aprendizaje, ya que muchas de las actividades que podemos encontrar pueden corregir sus errores y esto hace que tengan menos problemas de frustración y tengan ganas de superarse continuamente.

En definitiva, con el uso de las TIC hay una mejora de la comunicación y el lenguaje, para de esta forma puedan expresar sus emociones e identificar las de los demás, de manera que habrá una mejoría 
en las relaciones interpersonales, dotándoles de herramientas que les ayude en su interacción social, mediante la integración en el entorno, siendo capaz de desarrollar sus capacidades y su autonomía. Para poder dar respuesta a este trastorno que es cada vez más fácil de encontrar en las aulas, debemos profundizar un poco en su definición, etiología y características.

\section{Marco teórico}

Primeramente, el trabajo se centrará en la definición del Trastorno de Espectro Autista (TEA), su evolución, las causas y las dificultades que encontramos en niños/as con ese trastorno. Posteriormente, veremos cómo se ha incorporado las TIC como recurso educativo en educación infantil.

\subsection{Breve historia del origen del Trastorno del Espectro Autista (TEA)}

En la reciente historia del TEA ha habido una gran evolución en lo que al concepto y al trastorno se refiere. Uno de los primeros en hablar del TEA es Kanner (1943) que afirmaba en su artículo que los Trastornos Autísticos del Contacto afectivo afectaban a un conjunto de características que no permitía una relación normal y fluida con el resto de personas. También decía que afectaba a la parte de la adquisición y uso del lenguaje y en la necesidad que tenían estas personas de mantener su entorno sin variaciones. Y que podía darse o no otra serie de características como pueden ser el trastorno del sueño, problemas en la conducta, en la alimentación, etc.

Posteriormente, Asperger (1944) en su estudio Psicopatía Autística en la Niñez, puede observar que una parte de las características eran semejantes a las nombradas por Kanner, salvo que se diferenciaban en una adecuación cognitiva y verbal.

En los siguientes 20 años se piensa que el Trastorno del Espectro Autista es más un problema emocional o afectivo que cognitivo. No es hasta los años 80 que no se abandona esta idea, y se pasa a considerar como un trastorno de personalidad o una psicosis infantil. Hasta que llega Wing (1981) y los resultados que obtiene su estudio son bastantes parecidos a los obtenidos por Asperger. Por ello existe una transición a una concepción holística que invita a pensar a pensar que hay más de un trastorno del desarrollo, que pueden ser o no autistas, pero que presentan la misma sintomatología.

\subsection{Definición del Trastorno del Espectro Autista}

La definición del espectro autista ha ido sufriendo bastantes cambios hasta llegar a la definición actual obtenida del (Manual Diagnóstico y Estadístico de los Trastornos Mentales) DSM-V (APA, 2013). 
Según el DSM-V el Trastorno del Espectro Autista (TEA) se define como una perturbación grave, persistente y generalizada que afecta a la capacidad de interacción social y la comunicación interpersonal. También presentan comportamientos o actividades restringidas y estereotipadas. Se presentan desde la infancia y no acompañan al nivel de desarrollo según su edad ni a su maduración. Lo cual hace que limiten o perjudiquen en las relaciones sociales.

Por lo que podemos observar una serie de indicadores que caracterizan el TEA como son:

- Fracaso en reciprocidad socio-emocional.

- Dificultad en la adquisición y uso del lenguaje, tanto verbal como no verbal.

- Déficits en el desarrollo mantenimiento y comprensión de las relaciones sociales.

- Comportamientos repetitivos y conductas ritualistas y compulsivas.

\subsection{Clasificación según el CIE-10-ES}

Debemos aclarar que el CIE-10-ES es la clasificación internacional de enfermedades en su $10^{\mathrm{a}}$ edición y en la versión en español, es publicada por la Organización Mundial de la Salud (OMS). Según su clasificación que ha ido variando con el paso de los años en el 2016 se estipuló la siguiente clasificación:

- Trastorno autista. Autismo infantil, psicosis infantil o síndrome de Kanner: Se caracteriza por un desarrollo anormal que se manifiesta antes de los tres años de edad. Además, su comportamiento anormal se caracteriza por que afecta a la interacción social y a la comunicación y la presencia de actividades repetitiva, estereotipadas y restrictivas. También en algunos casos pueden aparecer otros trastornos como son fobias, trastornos del sueño, de alimentación, agresividad y rabietas, etc.

- Síndrome de Rett. Este trastorno afecta mayoritariamente al sexo femenino y no se conoce su causa. Se da entre el séptimo mes y los dos años de edad, siempre se da antes de los 4 años y después de un desarrollo normal o que en apariencia parece normal para su edad. Las características principales que se dan son, hipotonía muscular, es decir, perdida de la musculatura del cuerpo cosa que causará que haya perdida en la movilidad y la motricidad tanto fina como gruesa. Y suele ir acompañada de la pérdida parcial o total del desarrollo del lenguaje. A causa de estas características, también podemos observar dificultad a la hora de respirar, babo, pérdida del control de esfínter, etc. Por último, decir que produce un retraso en el crecimiento de la cabeza.

- Otro tipo de trastorno es el desintegrativo infantil o Síndrome de Heller. Es un trastorno profundo del desarrollo que se caracteriza por el desarrollo normal o aparentemente normal durante los dos primeros años de edad y que posteriormente en cuestión de unos meses hay 
una pérdida de capacidades que ya estaban adquiridas y que afecta a diversas áreas. Tienen regresiones tanto a nivel de lenguaje, como en actividades lúdicas o en relaciones sociales e interacciones en su entorno.

- Síndrome de Asperger. Se caracteriza por tener déficits en la interacción social y por presentar estereotipias y movimientos repetitivos. En este caso no encontramos retraso en el lenguaje o en el desarrollo cognitivo, aunque encontramos un retraso en el desarrollo motriz, ya que podemos encontrar movimientos más torpes.

- Otros trastornos generalizados del desarrollo (TGD). En él se incluye la hiperactividad en los que suele ser grave y asociada a la discapacidad intelectual, en el que tienden a tener un cociente intelectual (CI) inferior a 50. Presentan movimientos repetitivos y estereotipias.

- Trastorno generalizado del desarrollo (TGD) no especificado. Se trata de un trastorno en el que se presentan dificultades en el desarrollo pero que no cumplen con el resto de criterios de diagnóstico como los que hemos especificado anteriormente.

Se caracteriza por una alteración grave y que se da en tres áreas del desarrollo, que son; las habilidades lingüísticas, las relaciones sociales y la conducta.

\section{Intervención educativa en niños y niñas con TEA}

Cuando hablamos de intervención educativa lo primero que tenemos que tener en cuenta es que "esta atención es un programa individual y personalizado, que debe ser desarrollado de forma global y coordinada desde distintos ámbitos (salud, educación, servicios sociales), y que debe trabajar al máximo los conceptos de globalidad, sistematización y planificación de la intervención, integrando en todo el proceso de atención a la familia, potenciando su participación activa en el mismo.” (Alcantud y Dolz, 2006). Para una buena intervención educativa es primordial la detección precoz, es decir, el equipo docente debe estar alerta ante cualquier tipo de ítem que dé la voz de alarma para detectar este tipo de trastorno para así de esta manera poder actuar de manera rápida y correcta según las necesidades de cada persona afectada, ya que como hemos podido ver anteriormente en la clasificación que se da en el CIE-10-ES y que en los centros educativos se dará unas pautas de intervención que permita el desarrollo integral del alumnado en cuestión. Para poder actuar de la manera más precisa posible es necesario que haya una coordinación entre el tutor o tutora del alumno o alumna en cuestión y que tenga el apoyo y el trabajo en equipo con el equipo de orientación educativa y psicopedagógica, que entre ellos podemos encontrar profesionales de distintos ámbitos como, por ejemplo; psicólogos, pedagogos, logopedas, maestros en audición y lenguaje, trabajadores sociales y en algunos casos Médicos. Los tutores o tutoras tienen que trabajar conjuntamente con el EOEP, manteniendo reuniones y llevando un plan de intervención educativa conjunto que tenga co- 
herencia y cohesión. También debe haber reuniones con el resto de profesorado para que tengan constancia de la metodología que se usara en el caso del alumnado con TEA y por último y no menos importante es necesario tener reuniones y una buena comunicación con las familias, ya que forman parte importante en el proceso de aprendizaje de sus hijos y se debe llevar una puesta en común y la misma línea educativa ya que de esta manera evitaremos que el niño o niña con TEA no sepa cómo actuar delante de diversas situaciones.

La intervención educativa en el aula se centrará en trabajar las áreas afectadas por este trastorno como son: la interacción social, la comunicación y lenguaje y el área cognitiva y de autonomía personal.

Para poder hacer esta intervención se usarán diferentes elementos como son:

- Los Sistemas Aumentativos y Alternativos de la Comunicación (SAAC): según el portal ARASAAC "son formas de expresión distintas al lenguaje hablado, que tienen como objetivo aumentar (aumentativos) y/o compensar (alternativos) las dificultades de comunicación y lenguaje de muchas personas con discapacidad".

- Pictogramas: son símbolos gráficos que se utilizan para facilitar el lenguaje en los niños y niñas con algún tipo de dificultad. Estos símbolos son esenciales en el trabajo realizado con alumnado con TEA, ya que la mayoría de los niños y niñas con dificultades de aprendizaje, y tienen más facilidad para comunicarse mediante imágenes, ya que tienen menor nivel de abstracción.

- Tecnologías de la información y comunicación (TIC): que es el uso de las nuevas tecnologías en los procesos de enseñanza - aprendizaje del alumnado.

\section{El uso de las tic en la intervención educativa en niños y niñas con TEA}

Visto que nos encontramos en una sociedad donde podemos observar que allá donde vayamos encontramos medios tecnológicos y que cada vez está más en auge su uso, no cabe duda de que "las nuevas tecnologías han introducido profundos cambios en nuestro entorno y en los modos de relacionarnos. La televisión, el teléfono móvil y el uso de Internet han abierto nuevas posibilidades de comunicación, ocio y formación" (Gutiérrez y Martorell, 2011, p. 174).

De esta irrupción exagerada de la tecnología en el mundo nace "Las Tecnologías de la Información y la Comunicación, también conocidas como TIC, son el conjunto de tecnologías desarrolladas para gestionar información y enviarla de un lugar a otro. Abarcan un abanico de soluciones muy amplio. Incluyen las tecnologías para almacenar información y recuperarla después, enviar y recibir información de un sitio a otro, o procesar información para poder calcular resultados y elaborar informes" (Servicios TIC, 2006).

$6 \cdot$ International Journal of New Education | No4 
También debemos tener en cuenta que "El uso de las TIC habitualmente requiere unos ciertos conocimientos o competencias de manejo que deben ser aprendidas” (Gutiérrez y Martorell, 2011, p. 174).

Por ello, ya que, tenemos este recurso no hay nada mejor que usarlo para fines como puede ser el proceso de enseñanza - aprendizaje dentro del aula. Como he podido observar en prácticas investigadoras y de formación prácticas, en diferentes centros de educación infantil tanto de primer ciclo como de segundo las TIC están totalmente integradas para su uso, ya que, con ellas podemos trabajar en todas las áreas y de manera diversa según lo objetivos que queramos conseguir.

Por ello vamos a unir el uso de las TIC con el TEA para "procurar crear nuevas escenografías de aprendizaje, no reproducir las tradicionales y ello pasa necesariamente para la transformación del rol del profesor y del estudiante” (García Guillén, Garrote Rojas \& Jiménez Fernández, 2016).

Otras investigaciones (Hardy, Ogden, Newman \& Cooper, 2002; Moore \& Taylor, 2000; Neale, Leonard \& Kerr, 2002) han destacado que las TIC ofrecen a este alumnado un entorno controlado, pues ayudan a estructurar y organizar el entorno de interacción del alumno con TEA al configurarse como un medio muy predictible que ofrece contingencias comprensibles para el alumno. Ya que visualmente tienen más facilidad para estructuras y organizar su entorno y su intervención y de esta manera tienen más facilidad y se sienten más seguros a la hora de comunicarse. Por otro lado el alumnado con TEA les resulta más atrayente las TIC ya que mediante ellas tienen mas autonomía y facilidad a la hora de hacer sus procesos de aprendizaje, evitando la frustración mediante la autocorrección de sus actividades y pudiendo interactuar con el resto del alumnado y profesorado.

Por eso las TIC ofrecen muchas posibilidades en el proceso de E-A ya que son flexibles, potencian entornos interactivos y escenarios diferentes a los que se pudieran dar en un aula, pudiendo obtener una gran cantidad de información que hará que el alumnado sea crítico y por ello fomentará la autonomía, la colaboración y el trabajo en equipo, eliminando barreras espacio temporales.

\section{Metodología}

La búsqueda bibliográfica se ha realizado de forma sistemática a través de diversas bases de datos, entre ellas podemos destacar Digibug que es la base de datos de la Biblioteca universitaria de Granada en la que se ha realizado una búsqueda en toda la biblioteca, es decir, incluyendo trabajos finales de grado, tesis doctorales y revistas, y introduciendo criterios en castellano y ingles. En ella los términos o palabras clave que se han usado para la búsqueda son Trastorno del Espectro Autista, TEA, Tecnología de la Información y Comunicación, TIC, ICT, autism, intervención educativa en niños con TEA. 
Posteriormente también se ha realizado una búsqueda en la base de datos Dialnet, en la que he realizado una búsqueda en los documentos que contiene con las palabras clave, las TIC, las TIC y el TEA.

También he podido encontrar a través de Dialnet la revista Edmetic que es una Revista de Educación Mediática y TIC, de la que he podido obtener información bastante útil para la realización del trabajo.

Por otro lado, también he obtenido información de libros como Trastornos del desarrollo infantil y de Atención a la diversidad en el aula de la educación infantil, que son libros de asignaturas que he cursado y han servido de gran ayuda para la realización de este trabajo ya que trata entre otros sobre el tema del que se habla aquí.

Con este trabajo se pretende conocer más que es el Trastorno del Espectro Autista que muchas veces no se conoce exactamente lo que es y la clasificación que tiene y a que áreas afecta y por otro lado ver para que podemos usar las TIC en este tipo de trastorno ya que las nuevas tecnologías han irrumpido con tanta fuerza en el mundo y en el ámbito de la educación como tratamos en este trabajo mediante una investigación cualitativa de los documentos seleccionados para hablar del tema.

\section{Conclusiones}

Como hemos podido comprobar a lo largo de este trabajo, se ha avanzado mucho en lo que al concepto de TEA se refiere ya que ha tenido una evolución histórica a lo largo de los años y se ha ido clasificando cada vez de manera más acertada, diferenciando así los tipos que hay y las características que tiene cada tipo, así de esta manera se puede actuar de una manera más efectiva en la intervención educativa. También hemos visto que las nuevas tecnologías han tenido una gran irrupción a nivel mundial, pero también en el ámbito educativo ya que podemos encontrar que en la mayoría de centros se usan las TIC, ya sea mediante ordenador o usando la pizarra digital, etc.

Por último, hemos visto que el uso de las TIC en alumnado con TEA es muy efectivo, ya que ayuda a su aprendizaje, haciendo que puedan comunicarse de una manera más sencilla para ellos y así vemos que se sienten más seguros y con más ganas de interactuar en el aula. De esta manera se pueden trabajar todas las carencias que tienen de una manera más atrayente y divertida, haciendo que participen activamente en su proceso de aprendizaje, evitando la frustración y haciendo que cada vez sean más autónomos.

Finalmente, consideramos que el uso de las TIC es un avance educativo y sobretodo usándolo con alumnado con TEA, ya que facilita el trabajo en el aula y que estas personas puedan aprender de una manera más ajustada y teniendo más facilidad que si tuvieran que seguir una clase que se le diera al resto de compañeros y compañeras, ya que este alumnado tiene unas características diferentes y por ello hay que actuar en base a las necesidades que tiene cada persona. 


\section{Referencias}

Alcantud, F.; Avila, V.; Martínez, R. y Romero, R. (2001) Estudio del Impacto de las Nuevas Tecnologías en las personas con discapacidad. IMSERSO. Servei de Publicacions de la Universitat de Valencia.

Alcantud, F. (2003) Las tecnologías de ayuda y los trastornos generalizados del desarrollo. En Alcantud F. (Ed) Intervención Psicoeducativa en niños con trasornos generalizados del desarrollo. Madrid: Piramide.

American Psychiatry Association (2014). Manual de diagnóstico y Estadístico de los Trastornos Mentales (DSM-V), 5 a edición. Madrid: Editorial Médica Panamericana.

Basil, C. (1994) Sistemas aumentativos y alternativos de comunicación. En J. Peña (Ed) Manual de Logopedia. Barcelona: Masson.

Cuesta, J.L., y Abella, V. (2012). Tecnologías de la información y comunicación: aplicaciones en el ámbito de los trastornos del espectro del autismo. Revista Española sobre Discapacidad Intelectual, 43 (2), 6-25.

García Guillén, Susana, Garrote Rojas, Daniel y Jiménez Fernández, Sara (2016). Uso de las TIC en el Trastorno de Espectro Autista: aplicaciones. EDMETIC, Revista de Educación Mediática y TIC, 5(2), 134-157.

Gutiérrez, Pedro y Martorell, Almudena, (2011). Las personas con discapacidad intelectual ante las TIC. DOI:10.3916/C36-2011-03-09

Hardy, C., Ogden, J., Newman, J. y Cooper, S. (2002). Autism and ICT: A guide for teachers and parents. London: David Fulton.

Kanner, L. (1943). Autistic disturbances of affective contact. Nervous child, 2, 217-250. Recuperado de http://psycnet.apa.org/record/1943-03624-001

López Justicia M. D. y Polo Sánchez M. T. (2014). Trastornos del desarrollo infantil. (pp. 119-136). Madrid: Pirámide.

Lozano, J., Ballesta, F., Cerezo, M. C. y Alcaraz, S. (2013). Las Tecnologías de la Información y la Comunicación (TIC) en el proceso de enseñanza y aprendizaje del alumnado con Trastorno del Espectro Autista (TEA). Revista Fuentes, 14, 193-208. Recuperado de https:// www.uco.es/servicios/ucopress/ojs/index.php/edmetic/article/view/5780 
Mulas, F., Ros, G., Millá, M., Etchepareborda, M., Abad, L. y Téllez, M. (2010). Modelos de intervención en niños con autismo. Revista de Neurología, 3(50), 77-84. Recuperado de https://eoeptgdbadajoz.educarex.es/wp-content/ uploads/2012/12/Modelos-deintervenci\%C3\%B3n-en-ni\%C3\%B1os-peque\%C3\%B1os-con-autismo.pdf

Pérez de la Maza, L. (Noviembre 2000). Aplicaciones informáticas para alumnos/as con Trastornos del Espectro Autista y otros TGD. Abriendo puertas. Congreso llevado a cabo en el $\mathrm{X}$ Congreso nacional de autismo, Vigo, España.

Portal Aragonés de la Comunicación Aumentativa y Alternativa (s.f.). ¿Qué son los Sistemas Aumentativos y Alternativos de la Comunicación? Recuperado de: http://arasaac.org/index.php

Psicoactiva. (2004-2019). Recuperado de https://www.psicoactiva.com/cie10/cie10 47/

Reina, L. (2016). Las TIC en la educación de niños con Trastornos del Espectro Autista (Trabajo de Fin de Grado). Universidad de La Laguna, Santa Cruz de Tenerife, España.

ServiciosTIC (2006). Definición de TIC. [ONLINE]. Recuperado de http://www.serviciostic.com/ $\underline{\text { las-tic/definicion-de-tic.html. }}$

Silva Sández, G. y Rodríguez Miranda, F. de P.(2018). Una mirada hacia las TIC en la educación de las personas con discapacidad y con Trastorno del espectro autista: Análisis temático y bibliográfico. EDMETIC, Revista de Educación Mediática y TIC, 7(1), 43-65 doi: https://doi.org/10.21071/edmetic.v7i1.10030

Tárraga-Mínguez, R. y Sanz-Cervera, P. (2018). ¿Qué estrategias de intervención funcionan en la educación de los niños con trastorno del espectro autista? Revisión de evidencias en la literatura científica. ReiDoCrea, 7, 279-287.

Terrazas, M., Sánchez, S. y Becerra, M.T. (2016). Las TIC como herramienta de apoyo para personas con Trastorno del Espectro Autista (TEA). Revista nacional e internacional de educación inclusiva, 9(2), 102-136. Recuperado de https://dialnet.unirioja.es/servlet/ articulo?codigo $=5600282$

Tortosa, F. (2004). Tecnologías de ayuda en personas con Trastornos del Espectro Autista: Guía para docentes. Murcia: CPR Murcia I. 
Unidad Técnica de Codificación CIE-10-ES Ministerio de Sanidad, Servicios Sociales e Igualdad. Manual de codificación CIE-10-ES DIAGNÓSTICOS. Recuperado de https:// www.mscbs.gob.es/estadEstudios/estadisticas/normalizacion/CIE10/UT MANUAL DIAG 2016 prov1.pdf

Vidal, J.R., y Uña, F. (2012). Autismo II. Conectando con el Autismo: aplicaciones informáticas en el ámbito de los Trastornos del Espectro Autista. Aplicación de las Tecnologías de la Información y las Comunicaciones en la vida diaria de las personas con discapacidad. A Coruña: Universidad A Coruña Servicio de Publicaciones. 
